\title{
MicroRNA-24-1-5p promotes malignant melanoma cell autophagy and apoptosis via regulating ubiquitin D
}

\author{
YUJUAN XIAO $^{1,2}$, QINGCHUN DIAO ${ }^{2}$, YANHUA LIANG ${ }^{3}$, YUSHENG PENG ${ }^{1}$ and KANG ZENG ${ }^{1}$ \\ ${ }^{1}$ Department of Dermatology, Nanfang Hospital, Southern Medical University, Guangzhou, Guangdong 510515; \\ ${ }^{2}$ Department of Dermatology, Chongqing Traditional Chinese Medicine Hospital, Chongqing 400011; ${ }^{3}$ Department of \\ Dermatology, Shenzhen Hospital, Southern Medical University, Shenzhen, Guangdong 518100, P.R. China
}

Received April 22, 2017; Accepted September 7, 2017

DOI: $10.3892 / \mathrm{mmr} .2017 .7614$

\begin{abstract}
The present study aimed to investigate the key roles and possible regulatory mechanism of microRNA (miR)-24-1-5p in regulating the autophagy, and apoptosis of malignant melanoma cells. The expression levels of miR-24-1-5p in malignant melanoma tissues were determined. Human melanoma A375 cells were transfected with miR-24-1-5p mimic and control. The effects of miR-24-1-5p overexpression on regulating the expressions of autophagy-related proteins [microtubule-associated protein 1A/1B-light chain 3 (LC3)-II, LC3-I and Beclin-1] and apoptosis-related proteins [apoptosis regulator $\mathrm{Bcl}-2$ (Bcl-2) and (BCL2 like 1) Bcl-xL] were investigated. The percentage of apoptotic cells in different transfected cells was detected. In addition, luciferase reporter assays were performed to confirm whether ubiquitin $\mathrm{D}$ (UBD) was a target of miR-24-1-5p. The effects of UBD silencing on autophagy and apoptosis were also investigated. The expression levels of janus kinase (JNK), phosphorylated (P)-JNK, Jun proto-oncogene AP-1 transcription factor subunit (c-Jun) and p-c-Jun were determined following the overexpression of miR-24-1-5p, and UBD. In comparison with adjacent normal tissues, miR-24-1-5p was significantly downregulated in malignant melanoma tissues. Overexpression of miR-24-1-5p significantly increased the levels of LC3-II/I ratio and Beclin-1 expression, and decreased the expression levels of Bcl-2 and Bcl-xL. Flow cytometry also showed that miR-24-1-5p overexpression promoted cell apoptosis. Moreover, UBD was confirmed as a direct target of miR-24-1-5p. Silencing of UBD promoted melanoma cell autophagy and apoptosis via
\end{abstract}

Correspondence to: Professor Kang Zeng, Department of Dermatology, Nanfang Hospital, Southern Medical University, 1838 North Guangzhou Avenue, Guangzhou, Guangdong 510515, P.R. China

E-mail: npfkzk@163.com

Key words: cell apoptosis, malignant melanoma, miR-24-1-5p, UBD, cell autophagy regulating the expression levels of related proteins. Besides, the levels of the p-JNK/JNK and p-c-Jun/Jun ratios were significantly increased following miR-24-1-5p overexpression, which were reversed following co-overexpression of miR-24-1-5p, and UBD. Overexpression of miR-24-1-5p may target UBD, and subsequently promote the autophagy and apoptosis of malignant melanoma cells through activation of the JNK signaling pathway.

\section{Introduction}

Malignant melanoma is the most common type of skin cancer that begins in the melanocytes $(1,2)$. The incidence of this disease is increasing yearly around the world (3). Malignant melanoma is characterized by a complex and heterogeneous etiology (4), which is still largely unknown. Surgical therapy is effective for localized disease, however, the median survival of patients with metastatic malignant melanoma is only 6-9 months after treatment with radiation and conventional chemotherapy drugs (5). In addition, the prognosis for metastatic melanoma is extremely poor due to highly resistance to radiation and chemotherapy drugs (6). Therefore, a better understanding of key mechanism underlying malignant melanoma facilitates to the development of therapeutic strategies.

MicroRNAs (miRNAs), small non-coding RNA molecules, are known to play a crucial role in regulating carcinogenesis in various cancers, including malignant melanoma $(7,8)$. Accumulating evidences have confirmed a complex crosstalk between apoptosis and autophagy in cancer development $(9,10)$. miRNAs are found to regulate autophagy and exhibit important roles in the crosstalk between apoptosis and autophagy in disease progression (11). For instance, upregulation of microRNA-21 can influence the apoptosis of melanocytic cells in malignant melanoma (12). Depletion of miR-638 induces cell apoptosis and autophagy in melanoma (13). Thus, identification of key miRNAs associated with apoptosis and autophagy of melanoma cells will have great significance in the therapy of melanoma. Recently, miR-24-1-5p (also known as $\mathrm{miR}-24-1^{*}$ ) is found to regulate high molecular weight Hyaluronan (hmw-Ha)-mediated enhancement of human pulmonary endothelial barrier and vascular integrity $(14,15)$. Importantly, miR-24-1-5p is reported to be downregulated in cutaneous malignant melanoma by means of microarray 
analysis of microRNA expression profiles (16). However, whether miR-24-1-5p contributes to the development of malignant melanoma via regulating cell apoptosis and autophagy is largely unknown.

In this study, the miR-24-1-5p expression in malignant melanoma tissues was investigated. The effects of miR-24-1-5p overexpression of on the expression levels of cell autophagyand apoptosis-related proteins were investigated. In addition, whether ubiquitin D (UBD) was a target of miR-24-1-5p was explored. Besides, the expression levels of janus kinase (JNK) pathway-related proteins were determined after overexpression of miR-24-1-5p and UBD. The objective of our study was to investigate the potential roles of $\mathrm{miR}-24-1-5 \mathrm{p}$ in regulating apoptosis and autophagy of malignant melanoma cells, thus to elucidate the possible regulatory mechanism of miR-24-1-5p in malignant melanoma.

\section{Materials and methods}

Specimen collection. A total of 77 malignant melanoma specimens stored in our hospital were enrolled in this study. None of the patients received preoperative anticancer treatment. Thereinto, 29 specimens were primary malignant melanoma, 31 specimens were metastatic malignant melanoma, and 17 specimens were malignant melanoma associated with lymph node metastasis. Specimens that collected from the patients, was diagnosed by pathological analysis, and none of the patients received preoperative anticancer treatment. Tumor tissues and their adjacent normal tissues were collected from clinically ongoing surgical specimens. These samples were then snap-frozen with liquid nitrogen, and stored at $-80^{\circ} \mathrm{C}$. This study was approved by our hospital's ethics committee and each patient provided their written informed consent.

Cell culture. Human melanoma cell line A375 was purchased from American Type Culture Colleciton (ATCC; Manassas, VA, USA). Then A375 cells were cultured in RPMI-1640 medium (Sigma-Aldrich; Merck KGaA, Darmstadt, Germany) supplemented with $10 \%$ fetal bovine serum (FBS; Gibco; Thermo Fisher Scientific, Inc., Waltham, MA, USA) at $37^{\circ} \mathrm{C}$ in a humidified incubator with $5 \% \mathrm{CO}_{2}$. The medium was replaced every 2 days during subculture.

Cell transfection. A375 cells were digested and plated in 6-well plates and incubated at $37^{\circ} \mathrm{C}$ in $5 \% \mathrm{CO}_{2}$ for $24 \mathrm{~h}$. After $80-90 \%$ confluence, cells were transfected with miR-24-1-5p mimics, mimic control, si-UBD, si-control, pcDNA3.1 vector, and pcDNA3.1-UBD using Lipofectamine RNAiMAX (Life Technologies; Thermo Fisher Scientific, Inc.) following the instructions of manufacturer.

Detection of apoptosis. Cell apoptosis was detected using flow cytometry after staining with Annexin V-FITC and propidium iodide (PI; Sigma-Aldrich; Merck KGaA). Briefly, cells were plated in dish and allowed to settle for $48 \mathrm{~h}$. Cells were then harvested, washed with ice-cold PBS and then double labelled with Annexin V-FITC and PI according to the protocol recommended by the manufacturer. The mixtures were then analyzed using the FACSCalibur flow cytometer (BD Biosciences, San Jose, CA, USA) equipped with FACStation running

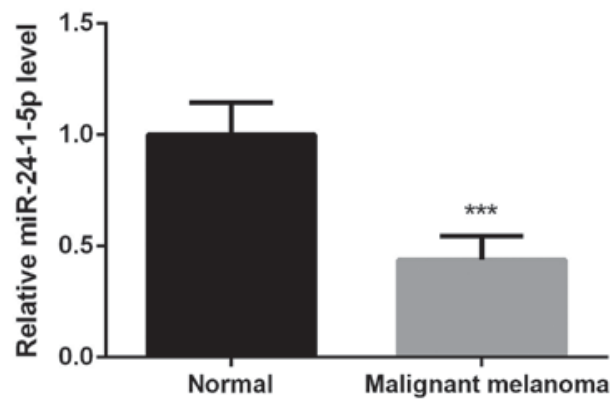

Figure 1. The expression levels of miR-24-1-5p in malignant melanoma tissues and their adjacent normal tissues. ${ }^{* * *} \mathrm{P}<0.001$.

CellQuest 3.0 software (BD Biosciences). Annexin V-FITC was used to determine the percentage of apoptotic, while PI was used to stain the dead cells.

Bioinformatic method and luciferase reporter assay. The target of miR-24-1-5p was predicted according to the information of TargetScanHuman database and UBD was predicted as a target of miR-24-1-5p. To further validate the predicted results, the wild type and mutant 3'UTR of UBD were cloned into the pGL3 Luciferase assays vector (Promega Corporation, Madison, WI, USA). The wild-type miR-24-1-5p contained binding sites of UBD 3'UTR with miR-24-1-5p. For luciferase assays, cells were plated in a 24-well plate and continued to incubate for another $24 \mathrm{~h}$. Cells were then co-transfected with Firefly luciferase constructs containing the 3'UTR wild-type or 3'UTR mutant of UBD, pRL-TK Renilla luciferase normalization control, miR-24-1-5p mimics or mimic control using Lipofectamine 2000 (Invitrogen; Thermo Fisher Scientific, Inc.). At $48 \mathrm{~h}$ after transfection, lysates were collected and measured with a Dual-Luciferase Reporter system (Promega Corporation) according to the protocols provided by the manufacturer. Renilla luciferase activity was used as an internal control.

Protein extraction and western blotting. Cells were lysed in whole cell lysis buffer, including $0.5 \mathrm{M}$ Tris- $\mathrm{HCl}$ ( $\mathrm{pH} 6.8), 1 \%$ b-mercaptoethanol, $0.02 \%$ bromophenol blue, $10 \%$ glycerol and $2 \%$ SDS. Then protein concentrations were detected using BCA protein assay kit (Pierce; Thermo Fisher Scientific, Inc.). Equal amount of protein sample was subjected to a $12 \%$ SDS-PAGE and then transferred to Hybond ECL membranes (Amersham Biosciences; GE Healthcare Life Sciences, Little Chalfont, UK). After being blocked with 5\% non-fat milk for $1 \mathrm{~h}$, the membranes were incubated with primary antibodies against UBD, JNK, p-JNK, c-Jun, p-c-Jun, light chain 3 (LC3), Beclin-1, Bcl-2, Bcl-xL and GAPDH (1:1,000 dilution; Cell Signaling Technology, Inc., Danvers, MA, USA) overnight at $4^{\circ} \mathrm{C}$. After that, the membranes were probed with appropriate horseradish-peroxidase conjugated secondary antibodies (1:2,000 dilution; Santa Cruz Biotechnology, Inc., Dallas, TX, USA) for $2 \mathrm{~h}$. After incubation with a chromogenic substrate, antibodyreactive proteins were visualized using the enhanced chemiluminescence (ECL) detection system (Thermo Fisher Scientific, Inc.). GAPDH was used as the internal control. 

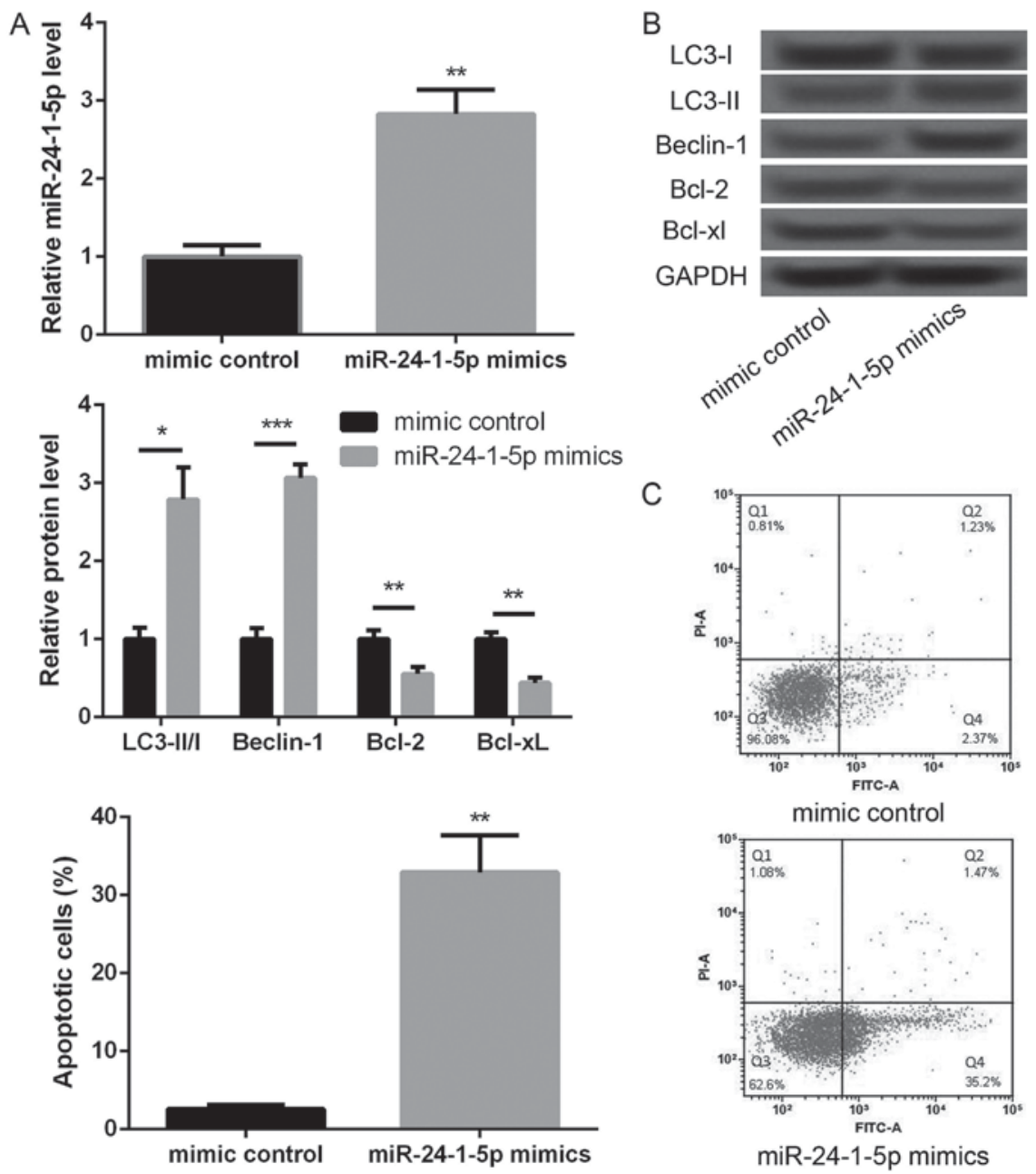

Figure 2. Overexpression of miR-24-1-5p promoted melanoma cell autophagy and apoptosis. (A) The expression levels of miR-24-1-5p in miR-24-1-5p mimic transfected cells and mimic control transfected cells. (B) The expression levels of LC3-I, LC3-II, Beclin-1, Bcl-2 and Bcl-xL in miR-24-1-5p mimic transfected cells and mimic control transfected cells. (C) Flow cytometry showed the percentage of apoptotic cells in different transfected cells. ${ }^{*} \mathrm{P}<0.05$, ${ }^{* *} \mathrm{P}<0.01$, ${ }_{* * * *} \mathrm{P}<0.001 . \mathrm{LC} 3$, light chain 3 .

Quantitative real time polymerase chain reaction ( $q R T-P C R)$. Total RNA was extracted from cultured cells by a modified method with TRIzol reagent (Invitrogen; Thermo Fisher Scientific, Inc.) according to the manufacturer's instructions. After detecting the quality of isolated RNA using a NanoDrop ${ }^{\circledR}$ ND-1000 UV-Vis spectrophotometer (Thermo Fisher Scientific, Inc.), reverse transcription into cDNA was then conducting using the PrimerScript First Strand cDNA Synthesis kit (Invitrogen; Thermo Fisher Scientific, Inc.). With a standard protocol recommended by the manufacturer, qRT-PCR was carried out using the SYBR ExScript qRT-PCR kit (Takara Biotechnology Co., Ltd., Dalian, China). Each reaction was performed in triplicate. The expressions of targets relative to $\beta$-actin was calculated using the comparative threshold $(\mathrm{Ct})$ cycle $\left(2^{-\Delta \Delta \mathrm{Ct}}\right)$ method.

Statistical analysis. Data from three independent experiments were expressed as mean \pm standard error of mean (SEM). Statistical significant differences were assessed between groups using the Student's t-test or one-way analysis of variance (ANOVA). A value of $\mathrm{P}<0.05$ was accepted as statistically significant. All statistical analyses were conducted with the
GraphPad Prism 5.0 software (GraphPad Software, Inc., La Jolla, CA, USA).

\section{Results}

miR-24-1-5p is downregulated in malignant melanoma tissues. We first detected the expression levels of miR-24-1-5p in malignant melanoma tissues and their adjacent normal tissues. The results showed that the expression levels of miR-24-1-5p in malignant melanoma tissues, including the primary malignant melanoma, metastatic malignant melanoma, and malignant melanoma specimens associated with lymph node metastasis were significantly downregulated in comparison with their adjacent normal tissues (Fig. 1, P<0.001). We then selected malignant melanoma A375 cells for the further analysis.

Overexpression of miR-24-1-5p promotes autophagy and apoptosis of melanoma A375 cells. To further investigate the effects of miR-24-1-5p, we overexpressed miR-24-1-5p in melanoma A375 cells. As shown in Fig. 2A, the expression of miR-24-1-5p was significantly increased in miR-24-1-5p mimic transfected cells compared with that in mimic control 

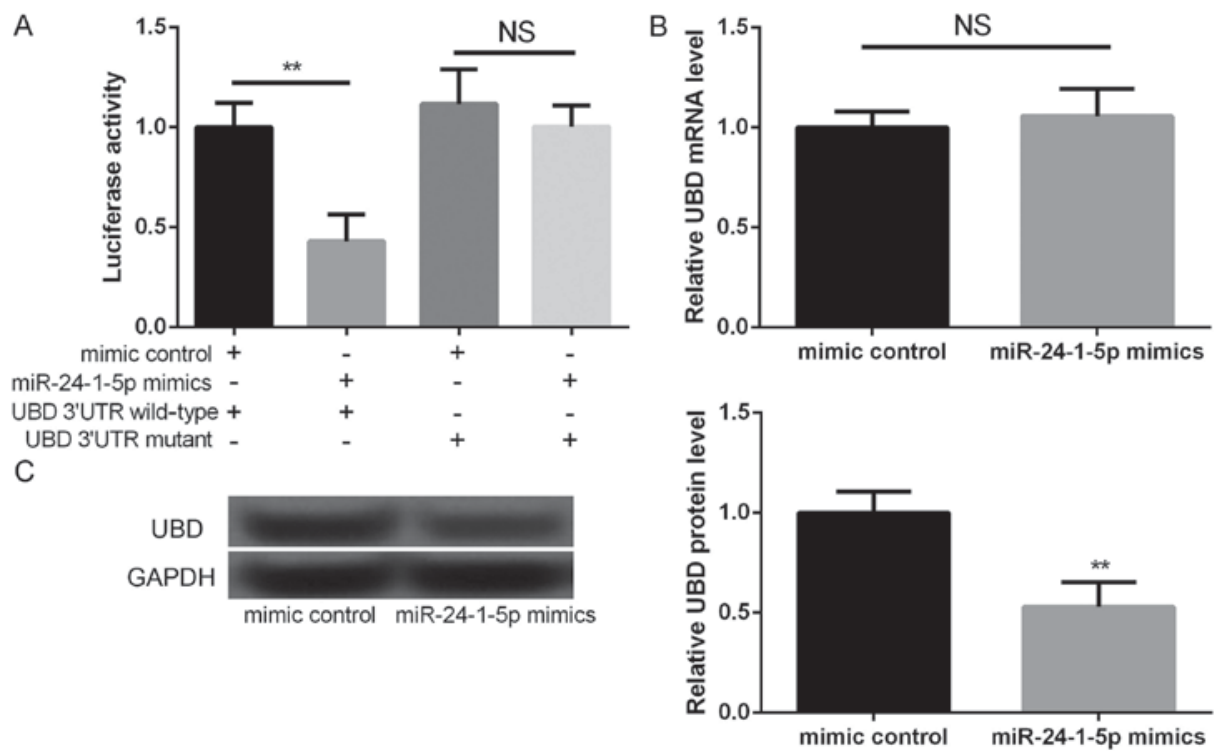

Figure 3. UBD was confirmed as a direct target of miR-24-1-5p. (A) Luciferase reporter analysis showed that miR-24-1-5p could target the wild-type 3'UTR of UBD. (B) The mRNA expression levels of miR-24-1-5p in miR-24-1-5p mimic transfected cells and mimic control transfected cells. (C) The protein expression levels of UBD in miR-24-1-5p mimic transfected cells and mimic control transfected cells. ${ }^{* *} \mathrm{P}<0.01$. UBD, ubiquitin D.

transfected cells $(\mathrm{P}<0.01)$, indicating that miR-24-1-5p was successfully overexpressed in melanoma A375 cell. We then further explore the effects of miR-24-1-5p overexpression on cell autophagy- and apoptosis-related proteins. In compared with mimic control group, the levels of LC3-II/I ratio and Beclin-1 expression were significantly increased in miR-24-1-5p mimic transfected cells (Fig. $2 \mathrm{~B}, \mathrm{P}<0.05$ ), indicating that miR-24-1-5p promoted melanoma cell autophagy. In addition, the protein expression levels of $\mathrm{Bcl}-2$ and $\mathrm{Bcl}-\mathrm{xL}$ were significantly decreased in miR-24-1-5p mimic transfected cells compared with mimic control transfected cells (Fig. 2B, P<0.05). The results of flow cytometry also showed that the percentage of apoptotic cells was significantly increased after overexpression of miR-24-1-5p (Fig. 2C, $\mathrm{P}<0.01)$.

$U B D$ is a direct target of miR-24-1-5p. According to the information of TargetScanHuman database, UBD was predicted as a potential target of miR-24-1-5p, which was further verified by luciferase reporter analysis. We found that the relative luciferase activities containing the wild-type 3'UTR of UBD were significantly decreased in miR-24-1-5p mimic transfected cells compared to that in mimic control transfected cells (Fig. 3A, $\mathrm{P}<0.01$ ), but not mutant 3'UTR of UBD. Moreover, although there is no significant different in the mRNA expression of UBD between miR-24-1-5p mimic transfection group and mimic control group (Fig. 3B), the protein expression of UBD was significantly decreased miR-24-1-5p mimic transfection group compared with mimic control group (Fig. $3 \mathrm{C}, \mathrm{P}<0.01$ ). These findings indicated that UBD was the direct target of miR-24-1-5p.

Silencing of UBD promotes autophagy and apoptosis of melanoma A375 cells. We further detected the expression of UBD in malignant melanoma tissues. The results showed that the expression of UBD in malignant melanoma tissues was significantly higher than that in their adjacent normal tissues (Fig. 4A and $\mathrm{B}, \mathrm{P}<0.05$ ). In addition, UBD was silenced to investigate whether UBD could regulate melanoma cell autophagy and apoptosis. As displayed in Fig. 4C, the protein expression of UBD in si-UBD group was significantly decreased compared with that in si-control (Fig. 4C, $\mathrm{P}<0.05$ ), suggesting that UBD was successfully silenced inmalignant melanoma cells. In addition, we found that after silencing of UBD, the levels of LC3-II/I ratio and Beclin-1 expression were significantly increased and the expression of Bcl-2 and Bcl-xL were markedly decreased (Fig. 4D, P $<0.05$ ). The results of flow cytometry also displayed an obviously increased apoptotic cells after silencing of UBD (Fig. 4E, P<0.05). These data indicated that silencing of UBD promoted melanoma cell autophagy and apoptosis significantly.

JNK pathway may be a potential mechanism involved in miR-24-1-5p-mediated autophagy and apoptosis in melanoma A375 cells. In previous study, activation of JNK pathway is shown to be involved in the induction of tumor cell autophagy and Apoptosis (17), thus we detected the expression changes of phosphorylated JNK and c-Jun after overexpression of miR-24-1-5p and UBD. As shown in Fig. 5, the levels of $\mathrm{p}-\mathrm{JNK} / \mathrm{JNK}$ ratio and $\mathrm{p}$-c-Jun/Jun ratio were significantly increased after miR-24-1-5p overexpression alone $(\mathrm{P}<0.05)$, indicating that overexpression of miR-24-1-5p could promote the activation of JNK pathway. However, the increased levels of $p-J N K / J N K$ ratio and $p$-c-Jun/Jun ratio were significantly reversed after overexpression of miR-24-1-5p and UBD $(\mathrm{P}<0.05)$. Moreover, overexpression of UBD could also significantly reverse the increased levels of LC3-II/I ratio and Beclin-1 expression, as well as the decreased expression of $\mathrm{Bcl}-2$ and $\mathrm{Bcl}-\mathrm{xL}(\mathrm{P}<0.05)$. These data imply that miR-24-1-5p may regulate the balance of apoptosis and autophagy via targeting UBD and involving in JNK pathway. 

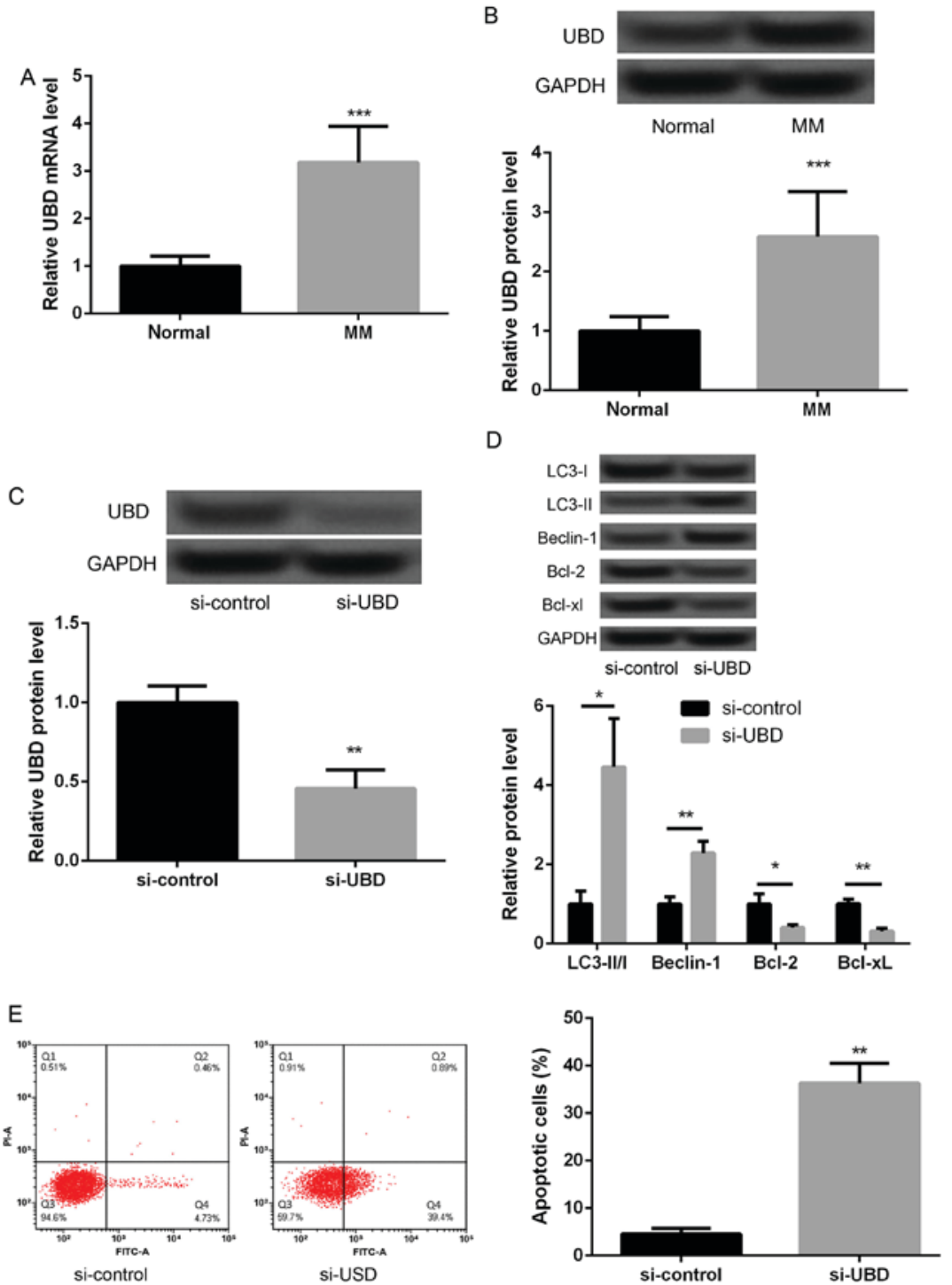

Figure 4. Silencing of UBD promoted melanoma cell autophagy and apoptosis. (A) The mRNA expression of UBD in malignant melanoma tissues and their adjacent normal tissues. (B) The protein expression of UBD in malignant melanoma tissues and their adjacent normal tissues. (C) The protein expression of UBD in si-UBD transfected cells and si-control transfected cells. (D) The expression levels of LC3-I, LC3-II, Beclin-1, Bcl-2 and Bcl-xL in different transfected cells. (E) Flow cytometry showed the percentage of apoptotic cells in different transfected cells. ${ }^{*} \mathrm{P}<0.05,{ }^{* * *} \mathrm{P}<0.01,{ }^{* * *} \mathrm{P}<0.001$. UBD, ubiquitin $\mathrm{D}$; LC3, light chain 3.

\section{Discussion}

In the present study, we found that miR-24-1-5p was significantly downregulated in malignant melanoma tissues, which was consistent with previous findings that miR-24-1 was a tumor suppressor and might serve as a disease progression marker in prostate cancer (16). Moreover, overexpression of miR-24-1-5p significantly increased the levels of LC3-II/I ratio and Beclin-1 expression, and decreased the expression levels of Bcl-2 and Bcl-xL. UBD was confirmed as a direct target of miR-24-1-5p. Silencing of UBD promoted melanoma cell autophagy and apoptosis via regulating the expressions of their related proteins. Besides, the levels of p-JNK/JNK ratio and p-c-Jun/Jun ratio were significantly increased after miR-24-1-5p overexpression alone, which were reversed after overexpression of miR-24-1-5p and UBD.

Apoptosis is the programmed cell death that maintains the balance of survival and death in living organisms $(18,19)$.
The expression levels of antiapoptotic Bcl-2 and Bcl-xL have been confirmed to increase with progression of malignant melanoma, which can reflect an increased malignant potential of metastatic melanoma cells after inhibiting cell apoptosis (20). Significantly, antisense-mediated inhibition of Bcl-2 is proved to sensitize malignant melanoma to apoptosis-inducing treatment modalities, and targeting Bcl-2 may be used as a highly effective strategy in the targeted therapy of malignant melanoma (21). In addition, autophagy is an evolutionarily conserved catabolic process which functions a dual role, playing pro-death or pro-survival role depending on the cell type and strength of specific stimuli $(22,23)$. Low levels of autophagy-regulated proteins LC3A and Beclin 1 are associated with an increased vascular density in cutaneous malignant melanoma (24). Autophagy genes Beclin 1 and MAP1LC3 are also confirmed to play a key role in tumor development in melanoma (25). Moreover, miR-204 can regulate hypoxia-reoxygenation-induced cardiomyocyte autophagy 

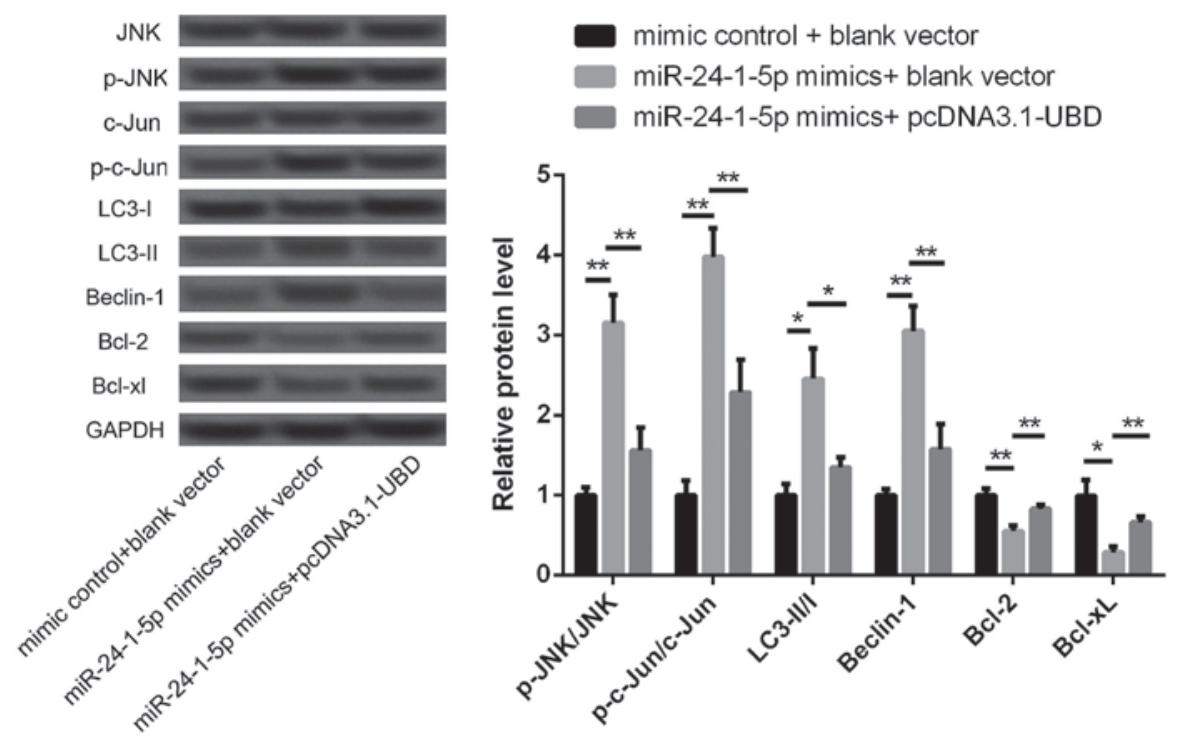

Figure 5. The expression levels of JNK, P-JNK, c-Jun, p-c-Jun, LC3-I, LC3-II, Beclin-1, Bcl-2 and Bcl-xL in different transfected cells. " $\mathrm{P}<0.05$, ${ }^{* *} \mathrm{P}<0.01$. JNK, janus kinase; P-JNK, phosphorylated -JNK; LC3, light chain 3; UBD, ubiquitin D.

via regulating LC3-II (26). In our study, miR-24-1-5p overexpression significantly increased the levels of autophagy-related proteins (LC3-II/I ratio and Beclin-1), and decreased the expression levels of apoptosis-related proteins (Bcl-2 and $\mathrm{Bcl}-\mathrm{xL}$ ). Although the roles of miR-24-1-5p in malignant melanoma have been fully explored, our results prompt that overexpression of miR-24-1-5p may promote cell autophagy and apoptosis in malignant melanoma.

Furthermore, UBD was confirmed as a direct target of miR-24-1-5p. In previous studies, UBD is found to play a crucial role in tumor development. For instance, increased expression of UBD in hepatic cancer cells is reported to have a growth advantage than cells without UBD expression (27). Overexpression of UBD is correlated with lymph node metastasis and TNM staging in gastric cancer, and its levels could be used as independent prognostic factors (28). Yan et al also demonstrated that UBD contributed to colon cancer progression and might serve as a novel prognostic indicator for predicting recurrence of stage II-III patients after curative surgery (29). Remarkably, UBD could regulates IRE1 $\alpha /$ JNK-dependent apoptosis in pancreatic $\beta$ cells (30). Activating of JNK and ERK is key mechanisms involved in Timosaponin AIII-induced apoptosis and autophagy in human melanoma A375-S2 cells (31). In our study, silencing of UBD promoted melanoma cell autophagy and apoptosis. Also, the levels of $\mathrm{p}-\mathrm{JNK} / \mathrm{JNK}$ ratio and p-c-Jun/Jun ratio were significantly increased after miR-24-1-5p overexpression alone, which were reversed after overexpression of miR-24-1-5p and UBD. Collectively, our data implying the regulatory relationship among miR-24-1-5p, UBD and JNK pathway in melanoma cell autophagy and apoptosis.

In conclusion, overexpression of miR-24-1-5p may target UBD and subsequently promote melanoma cell autophagy and apoptosis via activation of JNK pathway. miR-24-1-5p may function as a diagnostic marker or therapeutic target for malignant melanoma. However, we did not identify possible mechanisms related to the evolution of melanoma in different clinical stages, thus failing to evaluate if these changes were observed in the same patient or if it is an evolutionary effect of the melanoma. Exploration of the evolution of melanoma in different clinical stages is still needed to be performed in further investigations.

\section{References}

1. Gray-Schopfer V, Wellbrock C and Marais R: Melanoma biology and new targeted therapy. Nature 445: 851-857, 2007.

2. Luna JM, Hernández Guerrero A, Romero Méndez R and Luviano Ortiz JL: Solution of the inverse bio-heat transfer problem for a simplified dermatological application: Case of skin cancer. Ingenierí Mecáni Tecnolog Desarroll 4: 2014.

3. Little EG and Eide MJ: Update on the current state of melanoma incidence. Dermatol Clin 30: 355-361, 2012.

4. Bloethner S, Scherer D, Drechsel M, Hemminki K and Kumar R: Malignant melanoma-a genetic overview. Actas Dermosifiliogr 100 (Suppl 1): S38-S51, 2009.

5. Gogas HJ, Kirkwood JM and Sondak VK: Chemotherapy for metastatic melanoma: Time for a change? Cancer 109: 455-464, 2007.

6. Liu L, Kritsanida M, Magiatis P, Gaboriaud N, Wang Y, Wu J, Buettner R, Yang F, Nam S, Skaltsounis L and Jove R: A novel 7-bromoindirubin with potent anticancer activity suppresses survival of human melanoma cells associated with inhibition of STAT3 and Akt signaling. Cancer Biol Ther 13: 1255-1261, 2012.

7. Glud M and Gniadecki R: MicroRNAs in the pathogenesis of malignant melanoma. J Eur Acad Dermatol Venereol 27: 142-150, 2013.

8. Völler D, Ott C and Bosserhoff A: MicroRNAs in malignant melanoma. Clin Biochem 46: 909-917, 2013.

9. Hanahan D and Weinberg RA: Hallmarks of cancer: The next generation. Cell 144: 646-674, 2011.

10. Maiuri MC, Zalckvar E, Kimchi A and Kroemer G: Self-eating and self-killing: Crosstalk between autophagy and apoptosis. Nat Rev Mol Cell Biol 8: 741-752, 2007.

11. Xu J, Wang Y, Tan X and Jing H: MicroRNAs in autophagy and their emerging roles in crosstalk with apoptosis. Autophagy 8: $873-882,2012$.

12. Satzger I, Mattern A, Kuettler U, Weinspach D, Niebuhr M, Kapp A and Gutzmer R: microRNA-21 is upregulated in malignant melanoma and influences apoptosis of melanocytic cells. Exp Dermatol 21: 509-514, 2012.

13. Bhattacharya A, Schmitz U, Raatz Y, Schönherr M, Kottek T, Schauer M, Franz S, Saalbach A, Anderegg U, Wolkenhauer O, et al: miR-638 promotes melanoma metastasis and protects melanoma cells from apoptosis and autophagy. Oncotarget 6: 2966-2980, 2015. 
14. Singleton PA, Mirzapoiazova T, Mambetsariev N, Ge L, Mambetsariev B, Lennon FE, Poroyko V, Fang Y, Mutlu GM and Siddiqui S: miR-24-1-5p regulates high molecular weight Hyaluronan (hmw-Ha)-mediated enhancement of vascular Integrity: Role of foxa3. Am J Respir Crit Care Med 191: A2646, 2015.

15. Singleton PA, Siddiqui SS, Mirzapoiazova T, Mambetsariev N, Mambetsariev B and Lennon FE: MicroRNA Hsa-miR-24-1* regulates high molecular weight hyaluronan-mediated human pulmonary endothelial barrier enhancement. Am J Respir Crit Care Med 183: A4173, 2011.

16. Sand M, Skrygan M, Sand D, Georgas D, Gambichler T, Hahn SA, Altmeyer P and Bechara FG: Comparative microarray analysis of microRNA expression profiles in primary cutaneous malignant melanoma, cutaneous malignant melanoma metastases, and benign melanocytic nevi. Cell Tissue Res 351: 85-98, 2013.

17. Hooi WC: The role of ROS-mediated ERK and JNK activation in the induction of autophagy and apoptosis in tumour cells by a novel small molecule compound. Ph D, 2009.

18. Hassan M, Watari H, Abualmaaty A, Ohba Y and Sakuragi N: Apoptosis and molecular targeting therapy in cancer. Biomed Res Int 2014: 150845, 2014

19. Sankari SL, Masthan KM, Babu NA, Bhattacharjee T and Elumalai M: Apoptosis in cancer-an update. Asian Pac J Cancer Prev 13: 4873-4878, 2012.

20. Leiter U, Schmid RM, Kaskel P, Peter RU and Krähn G: Antiapoptotic bcl-2 and bcl-xL in advanced malignant melanoma. Arch Dermatol Res 292: 225-232, 2000.

21. Wacheck V, Losert D, Günsberg P, Vornlocher HP, Hadwiger P, Geick A, Pehamberger H, Müller M and Jansen B: Small interfering RNA targeting bcl-2 sensitizes malignant melanoma. Oligonucleotides 13: 393-400, 2003.

22. Maycotte $P$ and Thorburn A: Autophagy and cancer therapy. Cancer Biol Ther 11: 127-137, 2011.

23. Janku F, Mcconkey DJ, Hong DS and Kurzrock R: Autophagy as a target for anticancer therapy. Nat Rev Clin Oncol 8: 528-539, 2011.
24. Sivridis E, Koukourakis MI, Mendrinos SE, Karpouzis A, Fiska A, Kouskoukis C and Giatromanolaki A: Beclin-1 and LC3A expression in cutaneous malignant melanomas: A biphasic survival pattern for beclin-1. Melanoma Res 21: 188-195, 2011.

25. Yuan-Ting SU, Jin HL and Dermatology DO: The advances in Beclin 1 and MAP1LC3 in malignant melanoma. Chin J Dermatovenereol, 2014.

26. Jian X, Xiao-yan Z, Bin H, Yu-feng Z, Bo K, Zhi-nong W and Xin N: miR-204 regulate cardiomyocyte autophagy induced by hypoxia-reoxygenation through LC3-II. Int J Cardiol 148: 110-112, 2011.

27. Oliva J, Bardaggorce F, French BA, Li J, McPhaul L, Amidi F, Dedes J, Habibi A, Nguyen S and French SW: Fat10 is an epigenetic marker for liver preneoplasia in a drug-primed mouse model of tumorigenesis. Exp Mol Pathol 84: 102-112, 2008.

28. Ji F, Jin X, Jiao CH, Xu QW, Wang ZW and Chen YL: FAT10 level in human gastric cancer and its relation with mutant p53 level, lymph node metastasis and TNM staging. World J Gastroenterol 15: 2228-2233, 2009.

29. Yan DW, Li DW, Yang YX, Xia J, Wang XL, Zhou CZ, Fan JW, Wen YG, Sun HC, Wang Q, et al: Ubiquitin D is correlated with colon cancer progression and predicts recurrence for stage II-III disease after curative surgery. Br J Cancer 103: 961-969, 2010.

30. Brozzi F, Gerlo S, Grieco FA, Juusola M, Balhuizen A, Lievens S, Gysemans C, Bugliani M, Mathieu C, Marchetti P, et al: Ubiquitin D regulates IRE1 $\alpha / \mathrm{c}$-Jun $\mathrm{N}$-terminal kinase (JNK) protein-dependent apoptosis in pancreatic beta cells. J Biol Chem 291: 12040-12056, 2016.

31. Wang Y, Xu L, Lou LL, Song SJ, Yao GD, Ge MY, Hayashi T, Tashiro SI, Onodera S and Ikejima T: Timosaponin AIII induces apoptosis and autophagy in human melanoma A375-S2 cells. Arch Pharm Res 40: 69-78, 2017. 